\title{
SPIN-LADDERS WITH SPIN GAPS: A DESCRIPTION OF A CLASS OF CUPRATES
}

\author{
Sudha Gopalan, T. M. Rice, and M. Sigrist因 \\ Theoretische Physik, Eidgenössische Technische Hochschule - Hönggerberg, \\ CH-8093 Zürich, Switzerland. \\ (Received 17 November 1993.)
}

\begin{abstract}
We investigate the magnetic properties of the $\mathrm{Cu}-\mathrm{O}$ planes in stoichiometric $\mathrm{Sr}_{n-1} \mathrm{Cu}_{n+1} \mathrm{O}_{2 n}(\mathrm{n}=3,5,7, \ldots)$ which consist of $\mathrm{CuO}$ double chains periodically intergrown within the $\mathrm{CuO}_{2}$ planes. The double chains break up the two-dimensional antiferromagnetic planes into Heisenberg spin ladders with $n_{r}=\frac{1}{2}(n-1)$ rungs and $n_{l}=\frac{1}{2}(n+1)$ legs and described by the usual antiferromagnetic coupling $\mathrm{J}$ inside each ladder and a weak and frustrated interladder coupling $\mathrm{J}^{\prime}$. The resulting lattice is a new two-dimensional trellis lattice. We first examine the spin excitation spectra of isolated quasi one dimensional Heisenberg ladders which exhibit a gapless spectra when $n_{r}$ is even and $n_{l}$ is odd ( corresponding to $\mathrm{n}=5,9, \ldots$ ) and a gapped spectra when $n_{r}$ is odd and $n_{l}$ is even (corresponding to $\mathrm{n}=3,7, \ldots$ ). We use the bond operator representation of quantum $S=\frac{1}{2}$ spins in a mean field treatment with self-energy corrections and obtain a spin gap of $\approx \frac{1}{2} J$ for the simplest single rung ladder $(n=3)$, in agreement with numerical estimates. We also present results of the dynamical structure factor $\mathrm{S}(\mathrm{q}, \omega)$. The spin gap decreases considerably on increasing the width of the ladders. For a double ladder with four legs and three rungs $(n=7)$ we obtain a spin gap of only 0.1J. However,
\end{abstract}


a frustrated coupling, such as that of a trellis lattice, introduced between the double ladders leads to an enhancement of the gap. Thus stoichiometric $\mathrm{Sr}_{n-1} \mathrm{Cu}_{n+1} \mathrm{O}_{2 n}$ compounds with $\mathrm{n}=3,7,11, \ldots$ will be frustrated quantum antiferromagnets with a quantum disordered or spin-liquid ground state.

Typeset using REVTEX 


\section{INTRODUCTION}

The so-called "infinite layer" or "all layer" compound $\mathrm{SrCuO}_{2}$ represents a new family of the $\mathrm{Cu}-\mathrm{O}$ superconductors. This compound crystallizing in the tetragonal structure is characterized by an infinite stacking of $\mathrm{CuO}_{2}$ planes with intervening Sr layers without oxygen representing the simplest possible charge reservoir. The infinite layer $\mathrm{SrCuO}_{2}$ [1] are synthesized under extreme conditions involving high temperatures and high oxygen pressures and superconductivity in the $\mathrm{Cu}-\mathrm{O}$ layers is induced by modification of the intervening $\mathrm{Sr}$ layers. The $\mathrm{Cu}-\mathrm{O}$ layers can be either hole or electron doped depending on the dopants introduced in the $\mathrm{Sr}$ layer. The high pressure forms of $\mathrm{SrCuO}_{2}$ are however unstable above certain temperatures and a homologous series of oxides formulated as $\mathrm{Sr}_{n-1} \mathrm{Cu}_{n+1} \mathrm{O}_{2 n}$ (with $\mathrm{n}=3,5,7,9, \ldots .$.$) begins to be mixed in with the parent (\mathrm{n}=\infty)$ phase.

The homologous series of $\mathrm{Cu}$-rich high pressure phases $\mathrm{Sr}_{n-1} \mathrm{Cu}_{n+1} \mathrm{O}_{2 n}$ were recently studied [2] and were shown to consist of parallel lines of $\mathrm{CuO}$-double chains periodically intergrown within the $\mathrm{CuO}_{2}$ sheets as illustrated in Fig. 1(a). The occurrence of these double chains was interpreted as resulting from a periodic shear operation with a shear vector of $\frac{1}{2}\langle 110\rangle$ in the parent $(n=\infty)$ phase. This can also be visualised as the appearance of domain walls within the planes. The structure of one such sheet for a general $\mathrm{n}$ is shown in Fig. 1(a). The Cu-atoms are shown as big black dots while the oxygen atoms are located at all the points of intersections of the straight lines. The Sr atoms (not shown in the figure) are located at the centers of the squares which are empty and in planes displaced by $\pm \mathrm{c} / 2$ with respect to the copper-oxide sheet shown with $\mathrm{c}$ being the lattice constant perpendicular to the $\mathrm{Cu}-\mathrm{O}$ planes. The dotted circles are the square co-ordinated $\mathrm{Cu}$-atoms (like in the parent compound) and there are respectively $0,1,2, \ldots$ such $\mathrm{Cu}$-atoms in between two double chains for $\mathrm{n}=3,5,7 \ldots$ Thus the introduction of the $\mathrm{CuO}$ double chains periodically in the matrix of square planar co-ordinated $\mathrm{CuO}_{2}$ produces a superlattice geometry with unit cell parameters (na $\times a)$.

The double chains in $\mathrm{Sr}_{n-1} \mathrm{Cu}_{n+1} \mathrm{O}_{2 n}$ compounds affect dramatically the magnetic properties of the copper-oxide planes. In the stoichiometric compounds the $\mathrm{Cu}$ sites are singly occupied (in the hole notation) and the O-sites are empty. This can be modelled by a $\mathrm{S}=1 / 2$ Heisenberg model on the new lattice, the trellis lattice, formed from the Cu-sites. The exchange interaction between $\mathrm{Cu}$-atoms which are both not located along the double chains is given by that of the bulk $(n=\infty)$ value of J. Along the double chains however, two $\mathrm{Cu}$-ions (such as $\mathrm{A}$ and $\mathrm{B}$ of Fig. 1(a)) are connected via an O-site by $90^{\circ}$ bonds which gives rise to a ferromagnetic exchange [3]. The superexchange path between these $\mathrm{Cu}$-ions is through two orthogonal O-orbitals and this introduces a Hund's rule contribution leading to a ferromagnetic exchange $\mathrm{J}^{\prime}(<0)$ given in perturbation theory by

$$
J^{\prime}=\frac{8 t_{p d}^{4}}{\Delta^{2}}\left[\frac{1}{E_{T}+2 \Delta}-\frac{1}{E_{S}+2 \Delta}\right]
$$


where $\mathrm{t}_{p d}$ is the nearest neighbor hopping in the plane between the $\mathrm{Cu} 3 \mathrm{~d}\left(\mathrm{x}^{2}-\mathrm{y}^{2}\right)$ to the O2p $(\mathrm{x}, \mathrm{y})$ orbitals, $\Delta=\epsilon_{p}-\epsilon_{d}$ with $\epsilon_{p}$ and $\epsilon_{d}$ being respectively the on-site energies of the $\mathrm{O} 2 \mathrm{p}(\mathrm{x}, \mathrm{y})$ and the $\mathrm{Cu} 3 \mathrm{~d}\left(\mathrm{x}^{2}-\mathrm{y}^{2}\right)$ levels, and $\mathrm{E}_{T}$ and $\mathrm{E}_{S}$ are respectively the triplet and singlet levels. Using the values of $\mathrm{E}_{T}=1.8 \mathrm{eV}$ and $\mathrm{E}_{S}=7.3 \mathrm{eV}$ obtained from the level splittings [4] assuming an onsite Coulomb repulsion of $\mathrm{U}_{p}=4 \mathrm{eV}$ [5] and taking the standard values of $t_{p d}=1.3 \mathrm{eV}$ and $\Delta=3.3 \mathrm{eV}$ [5] we obtain $J^{\prime} / \mathrm{J}=0.1-0.2$.

Thus the planes in $\mathrm{Sr}_{n-1} \mathrm{Cu}_{n+1} \mathrm{O}_{2 n}$ are broken up into Heisenberg ladders with a weak and frustrated interladder coupling as illustrated in Fig. 1(b). There are 0,1,2... vertical chains (separated by the width a) cutting the dashed region in Fig. 1(b) corresponding to $\mathrm{n}=3,5,7 \ldots$. The lattice that results is a trellis lattice consisting of individual ladders with $n_{r}=\frac{1}{2}(n-1)$ rungs and $n_{l}=\frac{1}{2}(n+1)$ legs and coupled to each other through zig-zag couplings $\mathrm{J}^{\prime}$. Since $\mathrm{J}^{\prime} \ll \mathrm{J}$ we can to a first approximation neglect $\mathrm{J}^{\prime}$ and divide the $\mathrm{Cu}-\mathrm{O}$ planes into independent sets of ladders with $n_{r}=1,2,3 \ldots$ rungs and $\mathrm{n}_{l}=2,3,4 \ldots$.legs corresponding to $\mathrm{n}=3,5,7 \ldots$. The compounds with odd $\mathrm{n}_{r}$ and even $\mathrm{n}_{l}$ (corresponding to $\mathrm{n}=3,7,11, \ldots$ ) and those with even $\mathrm{n}_{r}$ and odd $\mathrm{n}_{l}$ (corresponding to $\mathrm{n}=5,9,13, \ldots$ ) exhibit different spin excitation spectra, the former having a spin gap with a shortrange exponentially decaying magnetic correlation function while the latter are gapless with a longrange powerlaw decay of the correlation function (like in the case of a single chain corresponding to $n=1$ ).

It was argued in ref. [6] that $\mathrm{Sr}_{n-1} \mathrm{Cu}_{n+1} \mathrm{O}_{2 n}$ compounds with $\mathrm{n}=3,7,11 \ldots$ would be frustrated quantum antiferromagnets and spin liquids. This was motivated by the numerical studies [7], [8] on isolated Heisenberg single-rung antiferromagnetic ladders with couplings J which exhibited a gap of $\approx \frac{1}{2} J$. Here we will follow an alternate treatment in terms of bond operators formulated [9] to study two-dimensional dimerised Heisenberg systems. In section II we will emphasize some of the important features of these bond operaters and use them in a mean-field treatment to study the simplest single rung Heisenberg ladder with two legs. In section III we report the results of the calculations on the spin excitation spectrum, spin gap, ground state energy and the dynamical structure factor $S(k, \omega)$. We extend the calculations to double ladders and also to periodic ladders in section IV. We show that the spin gap decreases considerably on increasing the width (rungs) of the ladders. In section V we study the effect of frustration on two spin-ladders and show that any non-zero frustrated coupling leads to an enhancement of the gap. This important result points towards the stabilization of a spin-liquid ground state in a trellis lattice. Such lattices will be realizations of short range RVB (Resonating Valence Bond) ground states in a $S=\frac{1}{2}$ system [10].

\section{SINGLE SPIN-LADDER}

We investigate here the properties of a single spin- $\frac{1}{2}$ ladder, shown in Fig. 2(a) which is described by the standard Heisenberg antiferromagnetic model 


$$
H=J \sum_{i} \mathbf{S}_{l_{i}} \cdot \mathbf{S}_{r_{i}}+\lambda J \sum_{i, m=l, r} \mathbf{S}_{m_{i}} \cdot \mathbf{S}_{m_{i+1}},
$$

where we take $\mathrm{J}$ to be the strength of the interaction along the rungs(i) of the ladder and $\lambda \mathrm{J}$ the interaction along the legs of the ladder. $\mathbf{S}_{l_{i}}$ and $\mathbf{S}_{r_{i}}$ are respectively spin- $\frac{1}{2}$ operators at the left(l) and right(r) -hand sites on each rung $\mathrm{i}$ of the ladder. In the limit of $\lambda=0$ (strong- coupling limit) the Hamiltonian of Eq.(2.1) reduces to a sum over contributions from independent two spin rungs. Thus it would be natural to tackle this problem from the limit of singlet dimers placed on the rungs and then switch on the interaction between them. It will be seen later that this starting point leads to reasonable results even for the case of $\lambda=1$ which is of interest here. We follow the bond-operator representation of quantum $\mathrm{S}=\frac{1}{2}$ - spins introduced [9] to study specifically the properties of dimerized phases. We emphasize here the essential features of the bond operators.

We consider two $\mathrm{S}=\frac{1}{2}$ spins $\mathbf{S}_{l}$ and $\mathbf{S}_{r}$ placed on each rung. The Hilbert space is spanned by four states which can be combined to form the singlet $\mid s>$ and the three triplet $\mid t_{x}>$, $\mid t_{y}>$, and $\mid t_{z}>$-states defined as being created out of the vacuum $\mid 0>$ by the singlet and triplet creation operators

$$
\begin{aligned}
& \left|s>=s^{\dagger}\right| 0>=\frac{1}{\sqrt{2}}(|\uparrow \downarrow>-| \downarrow \uparrow>), \\
& \left|t_{x}>=t_{x}^{\dagger}\right| 0>=-\frac{1}{\sqrt{2}}(|\uparrow \uparrow>-| \downarrow \downarrow>), \\
& \left|t_{y}>=t_{y}^{\dagger}\right| 0>=\frac{i}{\sqrt{2}}(|\uparrow \uparrow>+| \downarrow \downarrow>), \\
& \left|t_{z}>=t_{z}^{\dagger}\right| 0>=\frac{1}{\sqrt{2}}(|\uparrow \downarrow>+| \downarrow \uparrow>) .
\end{aligned}
$$

A representation of the spins $\mathbf{S}_{l}$ and $\mathbf{S}_{r}$ in terms of these singlet and triplet operators is given by,

$$
\begin{aligned}
S_{l \alpha} & =\frac{1}{2}\left(s^{\dagger} t_{\alpha}+t_{\alpha}^{\dagger} s-i \epsilon_{\alpha \beta \gamma} t_{\beta}^{\dagger} t_{\gamma}\right), \\
S_{r \alpha} & =\frac{1}{2}\left(-s^{\dagger} t_{\alpha}-t_{\alpha}^{\dagger} s-i \epsilon_{\alpha \beta \gamma} t_{\beta}^{\dagger} t_{\gamma}\right),
\end{aligned}
$$

where $\alpha, \beta$, and $\gamma$ represent respectively the components along the $\mathrm{x}, \mathrm{y}$, and z-axes and $\epsilon$ is the Levi-Civita symbol representing the totally antisymmetric tensor. Henceforth, it is assumed that all repeated indices over $\alpha, \beta$, and $\gamma$ are summed over.

A constraint of the form,

$$
s^{\dagger} s+t_{\alpha}^{\dagger} t_{\alpha}=1
$$

is introduced for each dimer in order to restrict the physical states to either singlets or triplets. Taking the singlet and triplet operators at each site to satisfy the bosonic commutation relations, 


$$
\left[s, s^{\dagger}\right]=1,\left[t_{\alpha}, t_{\beta}^{\dagger}\right]=\delta_{\alpha \beta},\left[s, t_{\alpha}^{\dagger}\right]=0
$$

we can reproduce the $\mathrm{S}=\frac{1}{2}, \mathrm{SU}(2)$ algebra of the spins $\mathbf{S}_{l}$ and $\mathbf{S}_{r}$ :

$$
\begin{gathered}
{\left[S_{m \alpha}, S_{m \beta}\right]=i \epsilon_{\alpha \beta \gamma} S_{m \gamma}, m=l, r,\left[S_{l \alpha}, S_{r \beta}\right]=0} \\
\vec{S}_{l} \cdot \vec{S}_{r}=-\frac{3}{4} s^{\dagger} s+\frac{1}{4} t_{\alpha}^{\dagger} t_{\alpha}, S_{l}^{2}=S_{r}^{2}=\frac{3}{4}
\end{gathered}
$$

Substituting the operator representation of spins defined in Eqs.(2.3) and (2.4) into the original Hamiltonian of Eq.(2.1) we obtain the following form:

$$
H=H_{0}+\lambda\left(H_{1}+H_{2}\right)
$$

where,

$$
\begin{aligned}
& H_{0}=\sum_{i} J\left(-\frac{3}{4} s_{i}^{\dagger} s_{i}+\frac{1}{4} t_{i \alpha}^{\dagger} t_{i \alpha}\right)-\sum_{i} \mu_{i}\left(s_{i}^{\dagger} s_{i}+t_{i \alpha}^{\dagger} t_{i \alpha}-1\right), \\
& \left.H_{1}=+\frac{J}{2} \sum_{i} t_{i \alpha}^{\dagger} t_{i+1 \alpha} s_{i+1}^{\dagger} s_{i}+t_{i \alpha}^{\dagger} t_{i+1 \alpha}^{\dagger} s_{i} s_{i+1}+h . c .\right), \\
& H_{2}=-\frac{J}{2} \sum_{i} \frac{1}{2}\left(1-\delta_{\alpha \beta}\right)\left(t_{i \alpha}^{\dagger} t_{i+1 \alpha}^{\dagger} t_{i \beta} t_{i+1 \beta}-t_{i \alpha}^{\dagger} t_{i+1 \beta}^{\dagger} t_{i+1 \alpha} t_{i \beta}+\text { h.c. }\right) .
\end{aligned}
$$

The part of the Hamiltonian containing triple " $t$ " operators vanishes identically in the present case due to reflection symmetry [11]. A site-dependent chemical potential $\mu_{i}$ is introduced to impose the constraint of Eq.(2.6). The Hamiltonian of Eq.(2.8) can now be solved by a mean-field decoupling of the quartic terms. This yields an effective Hamiltonian $H_{m R}$ with only quadratic operators. We take $\left.<s_{i}\right\rangle=\bar{s}$, which means that the "s" bosons are condensed. We replace the local constraint $\mu_{i}$ by a global one $\mu$ in accordance with the translational invariance of the problem along the ladder-axis ( $\hat{y}$-axis here). We will consider here only the terms $H_{0}$ and $H_{1}$ in Eq.(2.8) and will show later in the Appendix that inclusion of $\mathrm{H}_{2}$ changes the results only slightly. We perform a Fourier transformation of the operators $t_{i \alpha}^{\dagger}=\frac{1}{\sqrt{N}} \sum_{k} t_{k \alpha}^{\dagger} e^{i k r_{i}}$, where $\mathrm{N}$ is the number of dimers or rungs in the ladder and $\mathrm{k}$ is the wave-vector whose only non-zero component is along the ladder axis. Thus, retaining only terms $H_{0}$ and $H_{1}$ in Eq.(2.8) we obtain the mean-field limit,

$$
H_{m}(\mu, \bar{s})=N\left(-\frac{3}{4} J \bar{s}^{2}-\mu \bar{s}^{2}+\mu\right)+\sum_{k}\left[\Lambda_{k} t_{k \alpha}^{\dagger} t_{k \alpha}+\Delta_{k}\left(t_{k \alpha}^{\dagger} t_{-k \alpha}^{\dagger}+t_{k \alpha} t_{-k \alpha}\right)\right]
$$

where,

$$
\begin{aligned}
\Lambda_{k} & =\frac{J}{4}-\mu+\lambda J \bar{s}^{2} \cos k \\
\Delta_{k} & =\frac{\lambda}{2} J \bar{s}^{2} \cos k
\end{aligned}
$$


We have taken the lattice constant to be unity. The ground state wave-function can be written in the form $\left|\phi>=C \exp \left[\sum_{i} \bar{s}_{i} s_{i}^{\dagger}-\sum_{k} b_{k} t_{k \alpha}^{\dagger} t_{-k \alpha}^{\dagger}\right]\right| 0>$. The above mean-field Hamiltonian Eq.(2.12) can be diagonalised by a Bogoliubov transformation into new Boson operators $\gamma_{k \alpha}$ given by,

$$
\gamma_{k \alpha}=\cosh \theta_{k} t_{k \alpha}+\sinh \theta_{k} t_{-k \alpha}^{\dagger}
$$

where the coefficients $\cosh \theta_{k}$ and $\sinh \theta_{k}$ obtained in terms of $\Lambda_{k}, \Delta_{k}$ and $\omega_{k}$ are given by,

$$
\begin{aligned}
\cosh ^{2} \theta_{k} & =\frac{1}{2}\left(\frac{\Lambda_{k}}{\omega_{k}}+1\right), \\
\sinh ^{2} \theta_{k} & =\frac{1}{2} \operatorname{sgn}\left(\Delta_{k}\right)\left(\frac{\Lambda_{k}}{\omega_{k}}-1\right) .
\end{aligned}
$$

We finally obtain,

$$
H_{m}(\mu, \bar{s})=N\left(-\frac{3}{4} J \bar{s}^{2}-\mu \bar{s}^{2}+\mu\right)-\frac{N}{2}\left(\frac{J}{4}-\mu\right)+\sum_{k} \omega_{k}\left(\gamma_{k \alpha}^{\dagger} \gamma_{k \alpha}+\frac{1}{2}\right)
$$

where,

$$
\omega_{k}=\left[\Lambda_{k}^{2}-\left(2 \Delta_{k}\right)^{2}\right]^{\frac{1}{2}}
$$

The parameters $\mu$ and $\bar{s}$ are determined by solving the saddle point equations;

$$
<\frac{\partial H_{m}}{\partial \mu}>=0,<\frac{\partial H_{m}}{\partial \bar{s}}>=0
$$

We obtain the following self-consistent eqations from Eq.(2.18) evaluated at $\mathrm{T}=0$.

$$
\begin{aligned}
& \left(\bar{s}^{2}-\frac{3}{2}\right)+\frac{1}{2 \pi}\left[\sqrt{1+d} \mathbf{E}\left(\sqrt{\frac{2 d}{1+d}}\right)+\frac{1}{\sqrt{1+d}} \mathbf{K}\left(\sqrt{\frac{2 d}{1+d}}\right)\right]=0, \\
& \left(\frac{3}{2}+2 \frac{\mu}{J}\right)+\frac{2 \lambda}{\pi d}\left[\frac{1}{\sqrt{1+d}} \mathbf{K}\left(\sqrt{\frac{2 d}{1+d}}\right)-\sqrt{1+d} \mathbf{E}\left(\sqrt{\frac{2 d}{1+d}}\right)\right]=0 .
\end{aligned}
$$

where $\mathbf{K}(\eta)$ and $\mathbf{E}(\eta)$ are respectively the complete elliptic integrals of the first and second kind of modulus $\eta$. The dimensionless parameter $\mathrm{d}$ is defined as

$$
d=\frac{2 \lambda \bar{s}^{2}}{\left(\frac{1}{4}-\frac{\mu}{J}\right)}
$$

\section{RESULTS}




\section{A. Spin-triplet spectrum}

For each $\lambda$, the self-consistent solutions of $\bar{s}$ and $\mu$ are obtained from Eqs.(2.19) and are used to determine the excitation spectrum of the system of a single ladder obtained from Eq.(2.17) as,

$$
\omega_{k}=J\left(\frac{1}{4}-\frac{\mu}{J}\right)[1+d \cos k]^{\frac{1}{2}}
$$

These quasiparticle excitations arising from the spin-triplet states of the spin-ladder form a band whose bandwidth is a function of $\lambda$. The band-minimum is at $k=\pi$ and in Fig. 2(b) we present plots of $\omega_{k} / \mathrm{J}$ (relative to the band minima) as a function of $\mathrm{k}$ for $\lambda=0.1,0.5$ and 1.0. We notice that the dispersion around the band minimum gets more linear with increasing $\lambda$ which is reminiscent of the case of a linear chain $(\lambda=\infty)$.

Recently extensive numerical calculations have been performed [12] on Heisenberg spin ladders using Lanczos techniques. The spin-triplet dispersion relations were obtained on a ladder with $2 \times 12$ sites and for various values of $\lambda$. The results of such a calculation are shown as filled circles in Fig. 2(b) and the agreement with the present work is very good. For very small values of $\lambda$ the spin-triplet excitation spectrum has a bandwidth of $2 \lambda \mathrm{J}$ which is in excellent agreement with those obtained from the Lanczos data and from the strong coupling expansions of Heisenberg ladders [12]. The dispersion relation of Eq.(3.1) can be parametrized by a spin-wave velocity which is given by $c_{s}=J\left(\frac{1}{4}-\frac{\mu}{J}\right)\left(\frac{d}{2}\right)^{\frac{1}{2}}$. The spin-wave velocity reduces to 0 as $\lambda \rightarrow 0$ and is $1.1 \mathrm{~J}$ for $\lambda=1.0$.

\section{B. Spin Gap}

If the excitation spectrum $\omega_{k}$ is real and positive everywhere in the Brillouin-zone then the system is in a magnetically disordered (spin-liquid) phase and has a spin gap given by,

$$
\Delta=J\left(\frac{1}{4}-\frac{\mu}{J}\right)[1-d]^{\frac{1}{2}} .
$$

This is indeed found to be the case for a single Heisenberg antiferromagnetic ladder. In Fig. 2(c) we present a plot (continuous line) of the spin gap (in units of J) as a function of $\lambda$ obtained numerically by solving the non-linear Eqs.(2.19) and then substituting the self-consistent solutions $\bar{s}$ and $\mu$ into Eq.(3.2). Alternatively, Eq(2.19) and Eq.(2.20) can be combined to give the following single equation for $\mathrm{d}$

$$
d=\lambda\left[3.0-\frac{2}{\pi} \frac{1}{\sqrt{1+d}} \mathbf{K}\left(\sqrt{\frac{2 d}{1+d}}\right)\right]
$$

which is easier to solve numerically. The solution of $d$ for a given $\lambda$ can be used in Eq.(2.19) to determine $\mu$ and the spin gap is then obtained from Eq.(3.2) 
We can analytically study the asymptotic behavior of the spin gap. For small values of $\lambda$ (and hence d) the elliptic integrals of Eq.(2.19) can be expanded in a power series as,

$$
\begin{aligned}
E(\eta) & =\frac{\pi}{2}\left(1-\frac{1}{4} \eta^{2}-\frac{3}{64} \eta^{4}-\cdots\right), \\
K(\eta) & =\frac{\pi}{2}\left(1+\frac{1}{4} \eta^{2}+\frac{9}{64} \eta^{4}-\cdots\right),
\end{aligned}
$$

and we obtain,

$$
\begin{aligned}
d & =2 \lambda\left[1-\frac{3}{8} \lambda^{2}+O\left(\lambda^{4}\right)\right], \\
\left(\frac{1}{4}-\frac{\mu}{J}\right) & =1+\frac{1}{4} \lambda^{2}+O\left(\lambda^{4}\right),
\end{aligned}
$$

and the spin gap is given by

$$
\Delta=J\left(1-\lambda-\frac{\lambda^{2}}{4}+\frac{\lambda^{3}}{8}+O\left(\lambda^{4}\right)\right)
$$

This is consistent with Fig. 2(c) which shows a linear drop of the spin gap $\Delta$ for small values of $\lambda$. As $\lambda$ gets larger the spin gap as shown in Fig. 2(c) deviates considerably from the linear behavior and does not exhibit any critical value for $\lambda$ where the gap vanishes. Thus starting from the limit of $\lambda=0$ with spin singlets on each rung of the ladder, we see that any finite coupling $\lambda$ along the legs of the ladder delocalizes the singlets on the rungs thereby reducing the magnitude of the gap but not closing it completely.

The spin gap seems to approach zero as $\lambda \rightarrow \infty$ which should be the right gapless limit corresponding to decoupled spin- $\frac{1}{2}$ chains. However, on closer inspection of Eq.(3.3) we notice that at $\lambda=\infty, \mathrm{d}$ is given by the solution of the equation $\frac{1}{\sqrt{1+d}} \mathbf{K}\left(\sqrt{\frac{2 d}{1+d}}\right)-\frac{3 \pi}{2}=0$. This gives a value of $d$ which is very close to 1 . In addition it can be shown using Eq.(2.19) that as $\lambda \rightarrow 1, \frac{\mu}{J}$ diverges as $\lambda \ln (1-d)$. Hence the spin gap given by Eq.(3.2) also diverges as $\lambda \rightarrow \infty$. In the present case the spin gaps start to increase for values of $\lambda \geq 3$ and the mean-field treatment ceases to be valid.

The Heisenberg ladder has also been extensively studied numerically [8], [12] using the Lanczos technique and the spin gaps have been determined on $2 \times \mathrm{N}$ ladders with $\mathrm{N}=4,6,8,10$, and 12. An extrapolation of these results to the bulk limit is shown (as filled circles) in the inset of Fig. 2(c) as a plot of $\frac{\Delta}{\lambda J}$ versus $\lambda^{-1}$ for values of $\lambda \leq 1$. The full curve in the same inset indicates results obtained from the present calculation. The agreement is found to be good only for small values of $\lambda$.

The deviation of the present spin gaps from those obtained numerically can be traced to the $\lambda^{2}$ terms in the expansion of the spin gap as obtained in Eq.(3.7). In Ref. [12] it was shown in a strong coupling expansion that the spin gap varied as $\Delta=J\left(1-\lambda+\frac{3}{4} \lambda^{2}\right)$ for small values of $\lambda$. The $\lambda^{2}$ term arises in the strong coupling expansion from short range effects as the two nearest neighbor singlets surrounding the rung which is excited to a triplet 
state are ineffective in contributing to the energy of spin-triplet excitations to $O\left(\lambda^{2}\right)$. Thus the positive co-efficient obtained for the $\lambda^{2}$-term shifts the spin gaps above the $1-\lambda$ line as $\lambda$ is increased in agreement with the numerical results (see filled circles in the inset of Fig. $(2 \mathrm{c}))$. However, in the present treatment a positive coefficient is obtained in the expansion

for $\left(\frac{1}{4}-\frac{\mu}{J}\right)$ (Eq.3.6) but the expansion of (1-d $)^{\frac{1}{2}}$ produces an overall negative co-efficient for the $\lambda^{2}$-terms which shifts the spin gaps below the $1-\lambda$ line as $\lambda$ is increased. This implies that for a finite $\lambda$ the singlet and triplet levels are not pushed apart enough due to the wrong treatment of the short-range effect in the present mean-field method. This arises from treating the local constraint to be valid only on the average. We have therefore added an additional self energy term of $O\left(\lambda^{2}\right)$ in the triplet levels of Eq.(2.9) which corrects for the neglect of short range effect in the present treatment. We use the self enrgy term $\beta \lambda^{2}$ with an optimum value of $\beta=0.7$ which gives reasonable values of the spin gaps for $\lambda \leq 1$ as shown by the dashed line in the inset of Fig. 2(c). It should be pointed out that this self energy correction does not modify the dispersion curves of the spin-triplets shown in Fig. $2(\mathrm{a})$, it merely shifts the positions of the minima at $k=\pi$.

\section{Ground State Energy}

The ground state energy obtained from Eq.(2.16) is given by (neglecting $\mathrm{H}_{2}$ term)

$$
\frac{E_{G}}{N J}=\left(-\frac{3}{4} \bar{s}^{2}-\tilde{\mu} \bar{s}^{2}+\tilde{\mu}\right)-\frac{1}{2}\left(\frac{1}{4}-\tilde{\mu}\right)+\frac{1}{\pi}\left(\frac{1}{4}-\tilde{\mu}\right)(1+d)^{\frac{1}{2}} \mathbf{E}\left(\sqrt{\frac{2 d}{1+d}}\right),
$$

where $\mathrm{d}$ is defined in Eq. $(2.20)$ and $\tilde{\mu}=\frac{\mu}{J}$.

In the limit of $\lambda \rightarrow 0$ the correct ground state energy $\frac{E_{G}}{N J}=-\frac{3}{4}$ is recovered. As $\lambda$ is switched on the ground state energy decreases and we present in Table I the values of $\frac{E_{G}}{2 N J}$ obtained from Eq.(3.8) for certain values of $\lambda$. As discusssed before for the spin gap the ground state energies have also been obtained numerically [12] on finite length ladders and the extrapolated values to the bulk limit is also presented in Table I. We observe that the energies obtained from the present mean-field treatment compare well with numerical estimates for $\lambda \leq 0.5$.

\section{Structure Factor}

Structure factors are important physical quantities which can be measured experimentally. The dynamic spin-structure factor is defined as

$$
S^{0, \pi}(q, \omega)=\int d t e^{i \omega t}<S_{q, z}^{0, \pi}(t) S_{-q, z}^{0, \pi}(0)>
$$


where $S_{q, \alpha}^{0, \pi}$ represents the Fourier transform of the $\alpha$-th component of the spin-operator combination along the rungs given by

$$
S_{q, \alpha}^{0, \pi}(t)=\sum_{i} e^{i \vec{q} \cdot \overrightarrow{r_{i}}}\left[S_{l_{i, \alpha}}(t) \pm S_{r_{i, \alpha}}(t)\right]
$$

where $\mathbf{S}_{l_{i}}$ and $\mathbf{S}_{r_{i}}$ are the two spin-operators at each rung i of the ladder as shown in Fig. $2(\mathrm{a})$. Using the representations of the spins $\mathbf{S}_{l}$ and $\mathbf{S}_{r}$ in terms of the singlet and triplet operators as defined in Eqs.(2.3)and (2.4) we obtain

$$
\begin{aligned}
& S_{l_{i, \alpha}}+S_{r_{i, \alpha}}=-i \epsilon_{\alpha \beta \gamma} t_{i \beta}^{\dagger} t_{i \gamma}, \\
& S_{l_{i, \alpha}}-S_{r_{i, \alpha}}=s_{i}^{\dagger} t_{i \alpha}+t_{i \alpha}^{\dagger} s_{i} .
\end{aligned}
$$

Substituting these in Eq.(3.10) and transforming the "t" operators to Bogoliubov operators defined in Eq.(2.14) we obtain the following expressions for the dynamic spin structure factors;

$$
\begin{array}{r}
S^{\pi}(q, \omega)=\frac{1}{3} \bar{s}^{2}\left(\cosh 2 \theta_{q}-\sinh 2 \theta_{q}\right)\left(n_{q}+\Theta(\omega)\right) \delta\left(\omega_{q}-|\omega|\right), \\
S^{0}(q, \omega)=\frac{1}{9} \sum_{k}\left\{\left[\cosh 2\left(\theta_{k+q}-\theta_{k}\right)+1\right] n_{k}\left(1+n_{k+q}\right) \delta\left(\omega_{k+q}-\omega_{k}-\omega\right)\right. \\
\left.+\frac{1}{2}\left[\cosh 2\left(\theta_{k+q}-\theta_{k}\right)-1\right]\left(n_{k}+\Theta(\omega)\right)\left(n_{k+q}+\Theta(\omega)\right) \delta\left(\omega_{k+q}+\omega_{k}-|\omega|\right)\right\},
\end{array}
$$

where, $\Theta(\omega)$ is a step function and $\mathrm{n}_{k}$ is the Bose occupation factor and $\cosh \theta_{k}$ and $\sinh \theta_{k}$ are defined in Eq.(2.14)

The first term of Eq.(3.13) represents the simultaneous emission and absorption of excitations and vanishes identically at $\mathrm{T}=0$ while the second term corresponds to creation or annihilation of two excitations. In Fig. 3 we present the results of the structure factors $S^{0, \pi}(q, \omega)$ as a function of $\omega$ for $\mathrm{q}=\pi$ and for $\lambda=1.0$ after applying the self-energy correction described at the end of section III(b). The dominant contribution to the structure factor comes from $S^{\pi}(q, \omega)$. The static structure factors $S_{s t}^{0, \pi}(q)=\int d \omega S^{0, \pi}(q, \omega)$ show peaks at the commensurate wavevector of $\mathrm{q}=\pi$. The spin-spin correlations in the present case decay exponentially at large distances with a correlation length given by,

$$
\xi=\frac{c_{s}}{\Delta}=\left[\frac{d}{2(1-d)}\right]^{\frac{1}{2}}
$$

where $c_{s}$ is the spin-wave velocity and $\Delta$ the spin gap and $\mathrm{d}$ is as defined in Eq.(2.20). We notice that $\xi \rightarrow 0$ as $\lambda \rightarrow 0$ and for $\lambda=1$ we obtain a value of $\xi=2.66$.

\section{DOUBLE AND PERIODIC ARRAY OF LADDERS}

We now consider the effects on the spin gap of increasing the number of rungs in the ladder. We first consider the case of a simply connected double rung ladder shown in Fig. 
4(a). The two spin-ladders (denoted by left(L) and right(R)) are connected with a strength of $\lambda^{\prime} \mathrm{J}$ and in each individual ladder we assume as before an interaction strength of $\mathrm{J}$ along the rungs and $\lambda \mathrm{J}$ along the legs of the ladders. The Hamiltonian of the system can be written as,

$$
H=\sum_{\ell}\left[\left(H_{o_{\ell}}+\lambda H_{1_{\ell}}\right)+\frac{1}{2} \lambda^{\prime} H_{1_{\ell}}^{\prime}\right]
$$

where, $\ell$ denotes the ladder index, Left(L) or Right(R), and

$$
\begin{aligned}
& H_{o_{\ell}}=J \sum_{i}\left(-\frac{3}{4} s_{i_{\ell}}^{\dagger} s_{i_{\ell}}+\frac{1}{4} t_{i_{\ell} \alpha}^{\dagger} t_{i_{\ell} \alpha}\right)-\sum_{i \in R_{\ell}} \mu_{i}\left(s_{i_{\ell}}^{\dagger} s_{i_{\ell}}+t_{i_{\ell} \alpha}^{\dagger} t_{i_{\ell} \alpha}-1\right), \\
& H_{1_{\ell}}=\frac{J}{2} \sum_{i}\left(t_{i_{\ell} \alpha}^{\dagger} t_{i+1_{\ell} \alpha} s_{i+1_{\ell}}^{\dagger} s_{i_{\ell}}+t_{i_{\ell} \alpha}^{\dagger} t_{i+1_{\ell} \alpha}^{\dagger} s_{i_{\ell}} s_{i+1_{\ell}}+h . c .\right), \\
& H_{1_{\ell}}^{\prime}=-\frac{J}{4} \sum_{i, \ell \neq \ell}\left(t_{i_{\ell} \alpha}^{\dagger} t_{i_{\ell} \ell^{\prime} \alpha} s_{i_{\ell}}^{\dagger} s_{i_{\ell^{\prime}}}+t_{i_{\ell} \alpha}^{\dagger} t_{i_{\ell^{\prime}} \alpha}^{\dagger} s_{i_{\ell}} s_{i_{\ell}}+h . c .\right) .
\end{aligned}
$$

Here, we have neglected terms of the form $\mathrm{H}_{2}$ of Eq.(2.11) as it is shown in the appendix not to change the results significantly in the parameter range $0 \leq \lambda \leq 1$, which is of interest here. It should be pointed out here that the Hamiltonian containing triple " $t$ " operators will not vanish here in the $\mathrm{H}^{\prime}$ part of the Hamiltonian connecting the two ladders as it did in the previous case along the ladder axis. However this will not give any contribution in the magnetically disordered phase when we take averages over the operators in the mean-field decoupling.

Replacing $\left\langle s_{i_{\ell}}\right\rangle=\bar{s}$ and $\mu_{i_{\ell}}=\mu$ in Eq.(4.2) and taking Fourier transforms of the t-operators we obtain

$$
\begin{aligned}
H_{m}(\mu, \bar{s}) & =2 N\left(-\frac{3}{4} J \bar{s}^{2}-\mu \bar{s}^{2}+\mu\right)+\sum_{k, \ell}\left[\Lambda_{k} t_{k_{\ell} \alpha}^{\dagger} t_{k_{\ell} \alpha}+\Delta_{k}\left(t_{k_{\ell} \alpha}^{\dagger} t_{-k_{\ell} \alpha}^{\dagger}\right.\right. \\
& \left.\left.+t_{k_{\ell} \alpha} t_{-k_{\ell} \alpha}\right)+\frac{\Gamma_{k}}{2} \sum_{\ell \prime \neq \ell}\left(2 t_{k_{\ell} \alpha}^{\dagger} t_{k_{\ell} \prime \alpha}+t_{k_{\ell} \alpha}^{\dagger} t_{-k_{\ell^{\prime} \alpha}}^{\dagger}+t_{k_{\ell} \alpha} t_{-k_{\ell^{\prime} \alpha} \alpha}\right)\right] .
\end{aligned}
$$

where $\Lambda_{k}$ and $\Delta_{k}$ are as defined in Eq. (2.13) and $\Gamma_{k}=-\frac{\lambda^{\prime}}{4} \bar{s}^{2} J$. We perform a Bogoliubov transformation into two new Boson operators defined in terms of the t-operators of the left and right hand ladders as

$$
\gamma_{1,2_{k \alpha}}=\frac{1}{\sqrt{2}}\left[\left(\cosh \theta_{k} t_{k_{L} \alpha}+\sinh \theta_{k} t_{-k_{L} \alpha}^{\dagger}\right) \pm\left(\cosh \theta_{k} t_{k_{R} \alpha}+\sinh \theta_{k} t_{-k_{R} \alpha}^{\dagger}\right)\right] .
$$

These are simply symmetric (bonding) and anti-symmetric (anti-bonding) combinations of the individual transformations in the left and right ladders. We can now diagonalise the Hamiltonian of Eq.(4.3) using this transformation and we obtain the following

$$
H_{m}(\mu, \bar{s})=2 N\left(-\frac{3}{4} J \bar{s}^{2}-\mu \bar{s}^{2}+\mu\right)-N\left(\frac{J}{4}-\mu\right)+\sum_{k, b=1,2}\left[\omega_{b_{k}}\left(\gamma_{b_{k \alpha}}^{\dagger} \gamma_{b_{k \alpha}}+\frac{1}{2}\right),\right.
$$


where $\omega_{1,2_{k}}$ is defined as,

$$
\omega_{1,2_{k}}=\left[\Lambda_{k}^{2}-\left(2 \Delta_{k}\right)^{2} \pm 2 \Gamma_{k}\left(\Lambda_{k}-2 \Delta_{k}\right)\right]^{\frac{1}{2}} .
$$

Thus the spin-triplet excitation spectrum of a double ladder with three rungs and four legs consists of two branches for each $\lambda$ and $\lambda^{\prime}$ representing the bonding and the anti-bonding states. The splitting of these two branches is governed by the transfer matrix proportional to $\Gamma_{k}$ which is in turn proportional to $\lambda^{\prime}$. As $\lambda^{\prime} \rightarrow 0$ the two branches collapse into a single branch of the one rung ladder described in section III(a). We have plotted in Fig. 4(b) the spin-triplet excitation spectrum for the double ladder with the value $\lambda=\lambda^{\prime}=1$ and after applying the self-energy correction in each ladder as described at the end of section III(b). For comparison we show in the same figure a plot (shown as the dashed curve) of the excitation spectrum of a single rung ladder $\left(\lambda^{\prime}=0\right)$.

Following the same procedure as described in section II we can write down the mean-field equations evaluated at $\mathrm{T}=0$ as

$$
\begin{aligned}
& \left(\bar{s}^{2}-\frac{3}{2}\right)=-\frac{1}{4} \int \frac{d k_{y}}{2 \pi}\left(\frac{1+\frac{d}{2} a_{+}}{\left[1+d a_{+}\right]^{\frac{1}{2}}}+\frac{1+\frac{d}{2} a_{-}}{\left[1+d a_{-}\right]^{\frac{1}{2}}}\right), \\
& \left(\frac{3}{2}+2 \frac{\mu}{J}\right)=\frac{\lambda}{2} \int \frac{d k_{y}}{2 \pi}\left(\frac{a_{+}}{\left[1+d a_{+}\right]^{\frac{1}{2}}}+\frac{a_{-}}{\left[1+d a_{-}\right]^{\frac{1}{2}}}\right),
\end{aligned}
$$

where $\mathrm{d}$ is defined in Eq.(2.20), and $a_{ \pm}$is given by

$$
a_{ \pm}=\cos k \pm \frac{\lambda^{\prime}}{4 \lambda}
$$

The expression for the spin gap is

$$
\Delta=J\left(\frac{1}{4}-\frac{\mu}{J}\right)\left[1-d\left(1+\frac{\lambda^{\prime}}{4 \lambda}\right)\right]^{\frac{1}{2}} .
$$

We have plotted in Fig. 4(c) the spin-gaps of the double ladder as a function of $\lambda^{\prime}$ for different values of $\lambda$. We obtain a value of $0.1 \mathrm{~J}$ for the spin gap for $\lambda=\lambda^{\prime}=1$ and we notice that for each $\lambda$ the spin gap reduces drastically with $\lambda^{\prime}$. This can be explained by the fact the singlets along the rungs of the two ladders can not only delocalize along the ladder axes via the $\lambda$-coupling but also across the ladders through the transfer matrix proportional to $\lambda^{\prime}$. This produces for any non-zero coupling of two single rung ladders $\left(\lambda^{\prime} \neq 0\right)$ a splitting in the excitation spectrum of the individual ladders into two branches, one above (the antibonding branch) and the other below (the bonding branch) as shown in Fig. 4(b). Since the spin gap is a measure of the minimum energy in the excitation spectrum (at $k=\pi$ ) and since one of the branches of the double ladder is always lower than that of a single ladder for any non-zero $\lambda^{\prime}$ we can conclude that the spin gap of a double ladder is lower than that of single ladder and the spin gap should progressively decrease on increasing the width of the ladder. 
In the same context it is worthwhile to consider a periodic array of ladders, with $\lambda=1$ in each ladder, and simply connected to one another by $\lambda^{\prime}$ and then to ask the question for what value of $\lambda^{\prime}$ should the spin gap disappear since it is known that the ground state of a two-dimensional antiferromagnet has longrange order. We can study the system of ladders arranged periodically within the formalism described previously and the self-consistent equations are given by

$$
\begin{aligned}
& \left(\bar{s}^{2}-\frac{3}{2}\right)=-\frac{1}{2} \iint \frac{d^{2} k}{(2 \pi)^{2}} \frac{1+\frac{d}{2} a}{[1+d a]^{\frac{1}{2}}} \\
& \left(\frac{3}{2}+2 \frac{\mu}{J}\right)=+\lambda \iint \frac{d^{2} k}{(2 \pi)^{2}} \frac{a}{[1+d a]^{\frac{1}{2}}}
\end{aligned}
$$

where $\mathbf{k}$ is now a two-dimensional wave-vector with components $k_{y}$ along the ladder-axis and $k_{x}$ across the ladders. The parameter $\mathrm{d}$ is define in Eq.(2.20) and $a$ is given by

$$
a=\cos k_{y}-\frac{\lambda^{\prime}}{2 \lambda} \cos k_{x}
$$

Combining the equations in Eq.(4.10) we can write down the following single mean field equation for $\mathrm{d}$ which is easier to solve numerically.

$$
\frac{d}{\lambda}=3.0-\iint \frac{d^{2} k}{(2 \pi)^{2}} \frac{1}{[1+d a]^{\frac{1}{2}}} .
$$

The excitation spectrum of the periodic system of ladders has a minimum at $\mathbf{k}=(\pi, \pi)$ and the the spin gap given by $\Delta=J\left(\frac{1}{4}-\frac{\mu}{J}\right)\left[1-d\left(1+\frac{\lambda^{\prime}}{2 \lambda}\right)\right]^{\frac{1}{2}}$ is plotted in Fig. 5 as a function of $\lambda^{\prime}$ with $\lambda$ set to 1.0. We have again applied the self-energy corrections on each individual ladders as described at the end of section III(b). We notice that the spin gap vanishes for a value of $\lambda^{\prime} \approx 0.25$. As described before for the double ladder the decrease in the spin gap with $\lambda^{\prime}$ is explained by the delocalization of the singlets across the ladders. In this case the transfer matrix connecting the ladders is twice as large as in the case of a double ladder thereby decreasing the spin gap with $\lambda^{\prime}$ even faster than that of a double ladder.

\section{FRUSTRATED DOUBLE LADDERS AND TRELLIS LATTICE}

We now consider the double ladder described in the previous section but connected to each other through zig-zag frustrated couplings $J^{\prime}=\lambda^{\prime} J$ as shown in Fig.1(b), where $\mathrm{J}$ is the standard rung coupling. We retain the Hamiltonian in the form of Eq.(4.2) but with $\mathrm{H}_{1 \ell}^{\prime}$ containing an additional term given by

$$
H^{\prime} \prime_{\ell}=-\frac{J}{4} \sum_{i, \ell \neq \ell}\left(t_{i_{\ell} \alpha}^{\dagger} t_{i+1_{\ell^{\prime} \alpha}} s_{i_{\ell}}^{\dagger} s_{i+1_{\ell^{\prime}}}+t_{i_{\ell} \alpha}^{\dagger} t_{i+1_{\ell^{\prime} \alpha}}^{\dagger} s_{i_{\ell}} s_{i+1_{\ell^{\prime}}}+\text { h.c. }\right),
$$


The mean field Hamiltonian and the excitation spectrum are those described by Eqs.(4.5) and (4.6) with $\Gamma_{k}$ replaced by a new form $\Gamma_{k}=-\frac{\lambda^{\prime}}{4} \bar{s}^{2} J(1+\cos k)$ We obtain the self-consistent equations of Eq.(4.7) but with $\mathrm{a}_{ \pm}$given by $a_{ \pm}=\cos k \pm \frac{\lambda^{\prime}}{4 \lambda}(1+\cos k)$

The excitation spectrum of the double ladder with a zig-zig frustrated coupling also consists of two branches like in the case of a simply connected double ladder. However, in the present case at the minimum position of the spectrum $(k=\pi)$ the two branches become degenerate. This can be explained by the fact that the singlets on two successive rungs of each ladder are completely out of phase and hence are not able to delocalise across the ladders through the zig-zag frustrated couplings $\lambda^{\prime}$. Thus the spin gap defined by the minimum value of the spectrum at $k=\pi$ is now given by,

$$
\Delta=J\left(\frac{1}{4}-\frac{\mu}{J}\right)[1-d]^{\frac{1}{2}}
$$

which does not contain the $\lambda^{\prime}$ term explicitly. Thus the spin gaps should not change much form the values obtained for a single ladder and this is indeed what is observed as shown by the dashed lines in Fig. 4(c). We have again applied the self-energy correction to each individual ladder. We notice from Fig. 4(c) that for each $\lambda$ the spin gap slightly increases with $\lambda^{\prime}$. This simply reflects the slight changes in the self-consistent solutions of $\mathrm{d}$ and $\mu$ with $\lambda^{\prime}$.

We can extend the results discussed above to the trellis lattice shown in Fig. 1(b). The excitation spectrum would now be a function of $\mathbf{k}$ with components $k_{y}$ along the ladder axis and $k_{x}$ across the ladders. At the point $k_{y}=\pi$ the spectrum would be dispersionless with $k_{x}$ as a result of destructive interference of successive rung-singlets along the ladder axis. Thus a frustrated coupling between the ladders in the trellis lattice will not affect the spin gaps of each individual ladder and hence it will only help in retaining the spin-liquid nature.

\section{CONCLUSIONS}

We have shown that the $\mathrm{CuO}$ double chains intergrown periodically in the $\mathrm{CuO}_{2}$ planes of the new series of infinite-layer compounds $\mathrm{Sr}_{n-1} \mathrm{Cu}_{n+1} \mathrm{O}_{2 n}(\mathrm{n}=3,5,7, \ldots)$ creates a new twodimensional spin-lattice, the trellis lattice. Such a lattice can be described by Heisenberg spin ladders with $n_{r}=\frac{1}{2}(n-1)$ rungs and $n_{l}=\frac{1}{2}(n+1)$ legs with the usual antiferromagnetic coupling $\mathrm{J}$ inside each ladder and a weak and frustrated interladder coupling $\mathrm{J}^{\prime} \ll \mathrm{J}$. On neglecting $\mathrm{J}^{\prime}$ the $\mathrm{Cu}-\mathrm{O}$ planes can be thought of as built up of independent quasi onedimensional ladders with odd $\mathrm{n}_{r}$ and even $\mathrm{n}_{l}$ (corresponding to $\mathrm{n}=3,7,11, \ldots$ ) and those with even $\mathrm{n}_{r}$ and odd $\mathrm{n}_{l}$ (corresponding to $\mathrm{n}=5,9,13, \ldots$ ) which exhibit different spin excitation spectra. The former are gapped with shortrange exponentially decaying magnetic correlation functions while the latter are gapless with a longrange powerlaw decay of the correlation functions. 
We have used the bond operator representation of quantum spins in a mean field treatment with self-energy correction to first study the excitations of the simplest single rung ladder with two legs corresponding to $\mathrm{n}=3$. We have obtained the spin-triplet dispersion with a minimum at $k=\pi$ and a spin-gap of $\left(\approx \frac{1}{2} J\right)$. These are in good agreement with numerical estimates [8], [12]. It should be pointed out that conventional spin-wave calculations applied to a ladder [13], [12] predict gapless excitations for all values of the couplings $\lambda J$ along the legs of the ladder, even for $\lambda=0$ which is clearly unphysical.

We have been able to extend the mean field treatment to double ladders and to a periodic array of ladders. We find that increasing the rungs of the ladder drastically reduces the spin gap. For a double ladder with four legs and three rungs corresponding to $n=7$ we obtain a spin-gap of only $0.1 \mathrm{~J}$. In a periodic array of ladders with intraladder coupling $\mathrm{J}$ we find that the spin gap vanishes for an interladder coupling of $\approx 0.25 \mathrm{~J}$.

We have also studied the effect of a frustrated coupling, such as that of a trellis lattice, introduced between two ladders. We find a slight enhancement in the spin gap. Extending the results to a trellis lattice we can show that the spin-liquid nature will be preserved. Thus stoichiometric $\mathrm{Sr}_{n-1} \mathrm{Cu}_{n+1} \mathrm{O}_{2 n}$ compounds with $\mathrm{n}=3,7,11 \ldots$ will be frustrated quantum antiferromagnets with a quantum disordered or spin-liquid ground state. The resulting trellis lattice will be a realization of the short range RVB ground state for a spin- $\frac{1}{2}$ system [10].

The implications of these results to the other high $\mathrm{T}_{c}$ compound can be conjectured as follows. Certain underdoped high $\mathrm{T}_{c}$ samples have been experimentally shown to have spin gaps and a theoretical description of this in terms of frustrated next nearest neigbour coupling in a two-dimensional antiferromagnetic lattice is obtained only with large and unphysical values of the coupling. However if the two dimensional $\mathrm{Cu}-\mathrm{O}$ planes were thought of having some microstructure (introduced upon doping) then the spin gaps could be explained quite naturally.

\section{ACKNOWLEDGMENTS}

We wish to thank R. Hlubina and H.Tsunetsugu for stimulating conversations. This work was supported by grants from the Swiss National Fonds.

\section{APPENDIX:}

The results presented in section III were obtained by neglecting the term $\mathrm{H}_{2}$ of Eq. (2.11) containing four triplet operators. Here we will study the changes on including this term in 
a similar mean field treatment as presented before. By taking quadratic decouplings of the operators in $\mathrm{H}_{2}$ and performing Fourier transformations we obtain,

$$
H_{2}=-\frac{\lambda J}{3}\left[\sum_{k} \cos _{y}\left[2 P t_{k \alpha}^{\dagger} t_{k \alpha}-Q\left(t_{k \alpha}^{\dagger} t_{-k \alpha}^{\dagger}+t_{k \alpha} t_{-k \alpha}\right)\right]-N\left(P^{2}-Q^{2}\right)\right],
$$

where $\lambda$ and $\mathrm{N}$ are as defined previously and $\mathrm{P}$ and $\mathrm{Q}$ are two new mean-fields defined as

$$
\begin{aligned}
& P=\left\langle t_{i \alpha}^{\dagger} t_{i+1 \alpha}>,\right. \\
& Q=\left\langle t_{i \alpha} t_{i+1 \alpha}>.\right.
\end{aligned}
$$

On including Eq.(A1) into Eq.(2.11) we obtain a mean field Hamiltonian similar to Eq.(2.12)

$$
\begin{aligned}
H_{m R}(\mu, \bar{s}, P, Q)= & \left.N\left(-\frac{3}{4} J \bar{s}^{2}-\mu \bar{s}^{2}+\mu\right)-\frac{N \lambda J}{3}\left(P^{2}-Q^{2}\right)\right] \\
& +\sum_{k}\left[\Lambda_{k} t_{k \alpha}^{\dagger} t_{k \alpha}+\Delta_{k}\left(t_{k \alpha}^{\dagger} t_{-k \alpha}^{\dagger}+t_{k \alpha} t_{-k \alpha}\right)\right]
\end{aligned}
$$

but with $\Lambda_{k}$ and $\Delta_{k}$ replaced by

$$
\begin{aligned}
& \Lambda_{k}=\frac{J}{4}-\mu+\lambda J \bar{s}^{2} \cos k-\frac{2 \lambda}{3} P J \cos k, \\
& \Delta_{k}=+\frac{\lambda}{2} J \bar{s}^{2} \cos k+\frac{\lambda}{3} Q J \cos k .
\end{aligned}
$$

We can diagonalise Eq.(A3) by a Bogoliubov transformation as described earlier and the spectrum is given by Eq.(2.17) but with $\Delta_{k}$ and $\Lambda_{k}$ replaced by their new forms. The parameters $\mu, \bar{s}, \mathrm{P}$ and $\mathrm{Q}$ are obtained by solving the saddle-point equations which reduce at $\mathrm{T}=0$ to the following equations;

$$
\begin{aligned}
\left(\bar{s}^{2}-\frac{3}{2}\right) & =-\int \frac{d k}{2 \pi} \frac{\Lambda_{k}}{2 \omega_{k}} \\
\left(\frac{3}{2}+2 \frac{\mu}{J}\right) & =\lambda \int \frac{d k}{2 \pi} \frac{\Lambda_{k}-2 \Delta_{k}}{\omega_{k}} \cos k \\
P & =-\int \frac{d k}{2 \pi} \frac{\Lambda_{k}}{2 \omega_{k}} \cos k \\
Q & =\int \frac{d k}{2 \pi} \frac{\Delta_{k}}{\omega_{k}} \cos k
\end{aligned}
$$

By numerically solving for the mean-field parameters the spin gap is obtained and is plotted in Fig. 2(b) (dashed lines) as a function of $\lambda$. We notice that the inclusion of $H_{2}$ does not change the results significantly even for $\lambda \approx 1$ 


\section{REFERENCES}

* Present address: Dept. of Physics, Massachusetts Institute of Technology, Cambridge, MA 02139.

[1] T. Siegrist, S. M. Zahurak, D. W. Murphy and R. S. Roth, Nature 334, 231 (1988).

[2] M. Takano, Z. Hiroi, M. Azuma, and Y. Takeda, Jap. J. of Appl. Phys. Series 73 (1992); and Z.Hiroi, M. Azuma, M.Takano and Y. Bando, J. of Solid State Chem. 95, 230 (1991).

[3] P. W. Anderson in Magnetism I, ed. G. T. Rado and H. Suhl, (1963).

[4] Handbook of Atomic Data, ed. S. Fraga, K. M. S. Saxena, and J. Karoqski, Elsevier p.195, (1976)

[5] M. Hybertsen, E. B. Stechel, M. Schlüter and D. R. Jennison, Phys. Rev. B41, 11068 (1990).

[6] T. M. Rice, S. Gopalan and M. Sigrist, Europhys. Lett.,23, 445 (1993).

[7] R. Hirsch, Diplomarbeit, Universität Köln (1988).

[8] E. Dagotto, J. Riera and D.Scalapino, Phys. Rev. B45, 5744 (1992).

[9] S. Sachdev and R. N. Bhatt, Phys. Rev. B 41, 9323 (1990).

[10] P. W. Anderson, Science, 235, 1196, (1987).

[11] The ladder possesses a reflection plane perpendicular to the rungs and passing through the centers of the rungs. The terms containing the triple "t" operators change sign under the reflection and hence vanish in the evaluation of Eq.(2.8).

[12] T. Barnes, E. Dagotto, J. Riera and E.S. Swanson, Phys. Rev. B47, 3196 (1993).

[13] N.Katoh and M.Imada, J.Phys.Soc.Japan (to be published) 


\section{FIGURES}

FIG. 1. (a) Schematic diagram of a single copper-oxide sheet in $\mathrm{Sr}_{n-1} \mathrm{Cu}_{n+1} \mathrm{O}_{2 n}$ showing the parallel lines of $\mathrm{CuO}$ double chains. The $\mathrm{Cu}$-atoms are shown as big black dots while the oxygen atoms are located at all the points of intersections of the straight lines. The dashed regions correspond to the usual square co-ordinated $\mathrm{CuO}_{2}$ regions. (b) The two-dimensional trellis lattice formed from the exchange couplings in a single copper-oxide plane of $\mathrm{Sr}_{n-1} \mathrm{Cu}_{n+1} \mathrm{O}_{2 n}$ at stoichiometry. The usual antiferromagnetic coupling $J$ describes each of the ladders having $\mathrm{n}_{r}=\frac{1}{2}(\mathrm{n}-1)$ rungs and $\mathrm{n}_{l}=\frac{1}{2}(\mathrm{n}+1)$ legs and the ladders are coupled to one another through weak and frustrated zig-zag couplings $\mathrm{J}^{\prime}$.

FIG. 2. (a) A single $\mathrm{S}=\frac{1}{2}$ Heisenberg antiferromagnetic ladder with couplings $\mathrm{J}$ along the rungs (i) and $\lambda \mathrm{J}$ along the left(l) and right(r) legs of the ladder. (b) The dispersions of the spin-triplet excited states of the ladder of (a) relative to the band minimum at $\mathrm{k}=\pi$ for several values of $\lambda$. The continuous curves are obtained from the present mean-field method and the filled circles are the Lanczos results of Ref.12 obtained on a $2 \times 12$ ladder. (c) The spin gap $\Delta$ (in units of J) as a function of $\lambda$ obtained from the present mean-field treatment (continuous curve). The dashed curve is a similar plot after including higher order terms as described in the Appendix. In the inset a plot of $\Delta / \lambda J$ versus $\lambda^{-1}$ is shown. Continuous curve is from the present treatment (without higher order terms) and the filled circles are the numerical results of Ref.8. The dashed line in the inset is obtained after including a self-energy correction [described at the end of section III(b)] to the present treatment.

FIG. 3. The dynamical structure factors $S^{0, \pi}(q, \omega)$ as a function of frequency $\omega$ for $\mathrm{q}=\pi$. 
FIG. 4. (a) A double Heisenberg ladder consisting of two single $\mathrm{S}=\frac{1}{2}$ antiferromagnetic ladders of Fig. 2(a) connected to each other through the couplings $\lambda^{\prime} J$. (b) The dispersions of the spin-triplet excited states (bonding and anti-bonding states of Eq.(4.6)) of the double ladder of (a) for $\lambda=\lambda^{\prime}=1.0$. The dashed curve is a similar plot for a single ladder $\left(\lambda^{\prime}=0\right)$ with $\lambda=1$. (c) The spin gap $\Delta$ (in units of $J$ ) as a function of $\lambda^{\prime}$ for several values of $\lambda$. The dashed curves are similar plots obtained for the case of a double ladder connected to each other through the zig-zag frustrated couplings $J^{\prime}=\lambda^{\prime} J$ of Fig. 1(b).

FIG. 5. The spin gap (in units of the rung coupling J) of a periodic arrangement of ladders as a function of the interladder coupling $\lambda^{\prime}$ and with an intraladder couling of $\lambda=1$. 


\section{TABLES}

TABLE I. Results of the ground state energy $\frac{E_{G}}{2 N J}$ of a Heisenberg ladder

\begin{tabular}{lcr}
\hline \hline$\lambda$ & & $\frac{E_{G}}{2 N J}$ \\
\cline { 2 - 3 } & Present work & Ref. [12] \\
\hline 0.5 & -0.394 & -0.43 \\
1.0 & -0.475 & -0.578 \\
\hline \hline
\end{tabular}

